\title{
Micro RNA-483 Elevates Nucleus Pulposus Cell Proliferation and Matrix Degradation in Intervertebral Disc Degeneration
}

\author{
M. G. DAI ${ }^{1}$ AND J. N. YU ${ }^{1 *}$ \\ The First Clinical Medical College of Zhejiang Chinese Medical University, No. 546, Binwen Road, Hangzhou, Zhejiang 310053, \\ ${ }^{1}$ Department of Chronic Wound Diagnosis and Treatment Center, Hangzhou Geriatric Hospital, No. 469, Shen Ban Road, \\ Hangzhou, Zhejiang 310022, China
}

Dal and Yu: Matrix degradation in intervertebral disc degeneration

\begin{abstract}
This study was to evaluate the function of miR-483 in the development of intervertebral disc degeneration in order to find a potential novel therapeutic target for intervertebral disc degeneration. MiR-483 expression was determined in nucleus pulposus tissue, cells from patients with intervertebral disc degeneration and controls using real-time PCR. Western blotting was performed to determine anticollagen II, matrix metalloproteinase-2, matrix metallopeptidase-9, cyclin A, cyclin D1 and glyceraldehyde-3-phosphate dehydrogenase expression. Nucleus pulposus cell growth was detected in a MTT assay. Cell cycle was measured following flow cytometric detection. The expression level of miR-483 was downregulated significantly in intervertebral disc degeneration nucleus pulposus samples. Besides, miR-483 expression was negatively associated with the Pfirrmann grade. Furthermore, overexpression of miR-483 could promote nucleus pulposus cell growth, enhance the anticollagen II expression as well as inhibit the matrix metalloproteinase-2 and 9 expression. In addition, miR-483 caused an increase in the proportion of S-phase of nucleus pulposus cells. Present study revealed a novel miRNA in the progression of intervertebral disc degeneration, which could serve as a potential therapeutic approach for intervertebral disc degeneration.
\end{abstract}

Key words: miR-483, nucleus pulposus, intervertebral disc degeneration

The high incidence of low back pain (LBP) leads to severe disability, which increases medical and economic costs over the world ${ }^{[1]}$. Unfortunately, the pathogenesis of LBP remains unclear, and intervertebral disc degeneration (IDD) is considered to be the main risk factor ${ }^{[2]}$. IDD is a pathological process that has is affected by many factors such as, environment, aging, genetics, vibration, mechanical load, immune response and inflammatory response, but the specific molecular pathogenesis is still unclear ${ }^{[3-5]}$. Recently, excessive apoptosis of nucleus pulposus (NP) cells, followed by loss of extracellular matrix (ECM) in the NP tissue have attracted the attention during the development of IDD $^{[6,7]}$. Thus, it is of great significance to explore the mechanism of ECM loss in order to find new treatments for IDD.

MicroRNAs (miRNAs) are endogenous, non-coding and small RNAs, which are involved in several cell processes, including cell proliferation, apoptosis, differentiation and participate in the development and progress of many diseases ${ }^{[8]}$. Recently, miRNAs have proven to play a vital role in IDD $^{[9,10]}$. In fact, microarray analysis indicated that $\mathrm{miR}-483$ is downregulated in IDD compared to the control disks ${ }^{[1]}$. However, the expression and role of miR-483 in IDD remains unknown. In the present study, an attempt was made to investigate the function of miR-483 in the development of IDD in order to search for a potential novel therapeutic target for IDD.

NP tissue samples were collected from 18 IDD patients, who underwent spinal fusion from June 2017 to March 2019 in the Zhejiang Chinese Medical University. NP tissues collected from 18 patients with scoliosis served as the control. Routine MRI was performed before surgery on each patient. T2 weighted images helped to determine the degree of disc degeneration according to modified Pfirrmann classification. The study was approved by the ethics committee of Zhejiang Chinese Medical University. All patients participated in the study have signed an informed consent form voluntarily. 
NP cells were isolated from patient disc surgical specimens and cultured in DuIbecco's modified eagle's medium (DMEM, Gibco, USA) containing $10 \%$ fetal bovine serum (FBS, Gibco), $100 \mathrm{U} / \mathrm{ml}$ penicillin, 100 $\mathrm{mg} / \mathrm{ml}$ streptomycin and $1 \% \mathrm{~L}$-glutamine, and were grown under $5 \% \mathrm{CO}_{2}$ in a humidified atmosphere at $37^{\circ}$. MiR-483 mimic and mimic NC were obtained from RiboBio (Guangzhou, China) and transfected to NP cells according to the instructions using lipofectamine-2000.

The NP cells were homogenized and the protein concentrations were determined using BCA protein assay kit (Beyotime, China). Primary antibodies antiCol II, antiMMP-2, antiMMP-9, anticyclin A, anticyclin D1 and antiGAPDH were purchased from Cell Signaling Technology (Beverly, USA). GAPDH was used as a loading control. Total RNA of NP samples and cells were extracted with TRIzol (Invitrogen) and cDNAs were synthesized using iScript cDNA Synthesis Kit (Bio-Rad, Hercules, CA, USA). The iQ5 RealTime PCR Detection System (Bio-Rad) was used to perform real-time PCR (RT-PCR) reactions. The sequences of primers were shown as following: miR483- forward 5'-GGCACCAGGGTTTAAT-3' and reverse 5'-CTCCTGTTAGGGCTGT-3'; U6-forward 5'-GCTTCGGCAGCACATATACTAAAAT-3' and reverse 5'-CGCTTCACGAATTTGCGTGTCAT-3'.

The NP cells were cultured in 96-well culture plate with complete culture medium. After incubation for specified time, $20 \mu \mathrm{l}$ MTT was added to each well, incubation was continued at $37^{\circ}$ for $4 \mathrm{~h}$, then the medium was removed and the precipitated formazan was dissolved in DMSO. Absorbance was measured at $450 \mathrm{~nm}$ on a microplate spectrophotometer (Biotek Instruments).

Flow cytometry FACSDiva 6.1.1 (Becton Dickinson) was used for measuring cell cycle. In brief, the cells

a

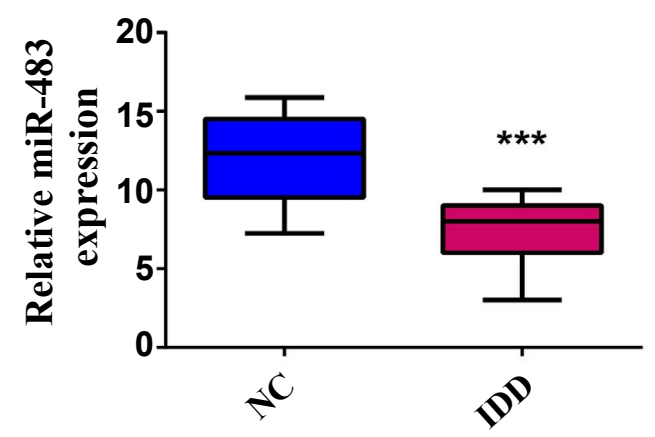

were collected and fixed with $70 \%$ ethanol at $-20^{\circ}$ overnight. The cells were mixed with $20 \mu \mathrm{g} / \mathrm{ml}$ RNaseA (Sigma-Aldrich, USA) and cultured with $50 \mu \mathrm{g} / \mathrm{ml}$ propidium iodide (Sigma-Aldrich, USA) at $37^{\circ}$ for 30 min according to the instructions.

SPSS 19.0 software (SPSS Inc., USA) was used for statistical analysis. The continuous variables were expressed as (mean $\pm \mathrm{SD})$. The t-test was used for comparison between two groups, and $\mathrm{p}$ value $<0.05$ was considered statistically significant. To explore the role of miR-483 in IDD, first its expression was determined in 18 normal NP and 18 degenerated NP samples using qRT-PCR. The results showed that the expression was significantly downregulated in IDD $\mathrm{NP}$ cases relative to normal subjects (fig. 1a). It was confirmed that MiR-483 expression level in IDD was negatively associated with the Pfirrmann grade. (fig. $1 \mathrm{~b}, \mathrm{r}=0.68, \mathrm{p}<0.05$ ). To determine the effect of miR-483 on NP cell growth, NP cells were transfected with miR-483 mimic and its control mimic, which showed the marked efficiency of miR-483 mimic on NP cells (fig. 2a). As displayed in fig. 2b, MTT assay showed that miR-483 overexpression promoted the $\mathrm{NP}$ cell growth. In addition, miR-483 overexpression enhanced the Col II expression level in NP cells (fig. 2c). It was subsequently confirmed that the MMP-2 and MMP-9 expression in the NP cells after transfected with miR-483 mimic or mimic NC. The western blot analysis demonstrated that overexpression of miR-483 inhibited the expression of MMP-2 and MMP-9 in NP cells (fig. 3). To further clarify the effect of miR-483 on NP cells, the effect of miR-483 on NP cell cycle was tested. The results of flow cytometry showed that miR483 could increase the proportion of $\mathrm{S}$ and $\mathrm{G} 2 / \mathrm{M}$ cells (fig. 4a). It is suggested that miR-483 could promote DNA synthesis and mitosis of NP cells. In addition,

b

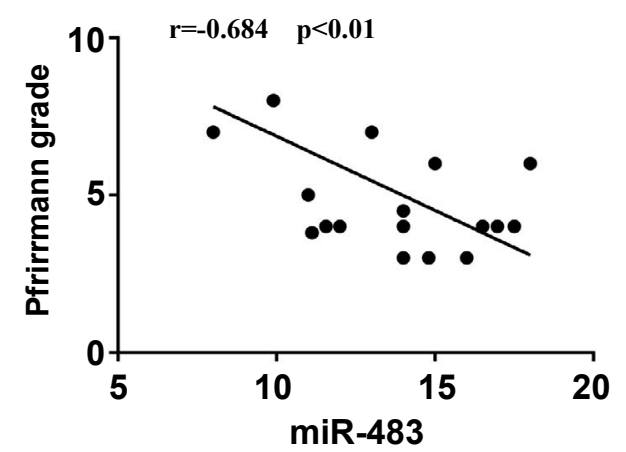

Fig. 1: MiR-483 expression level decreased significantly in IDD samples a. The miR-483 expression in 36 human NP tissues was detected by qRT-PCR. U6 was used as the internal control. b. miR-483 expression level was negatively related with the Pfirrmann scores. ***p<0.05. 
a

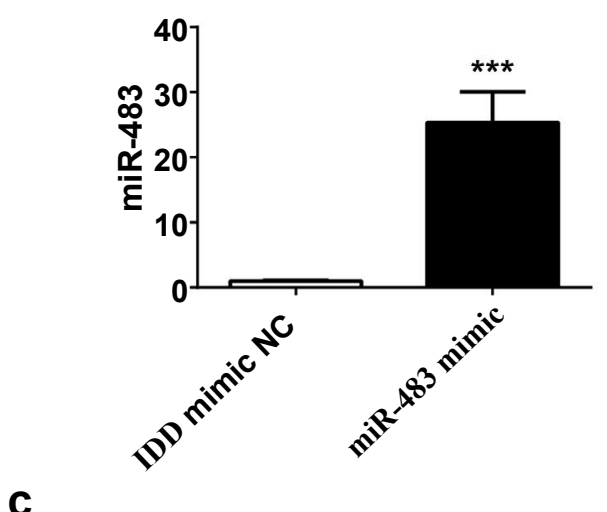

b

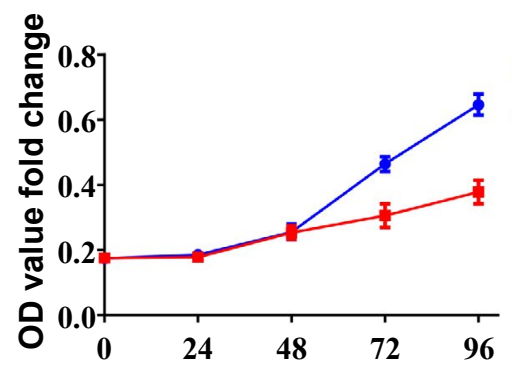

d

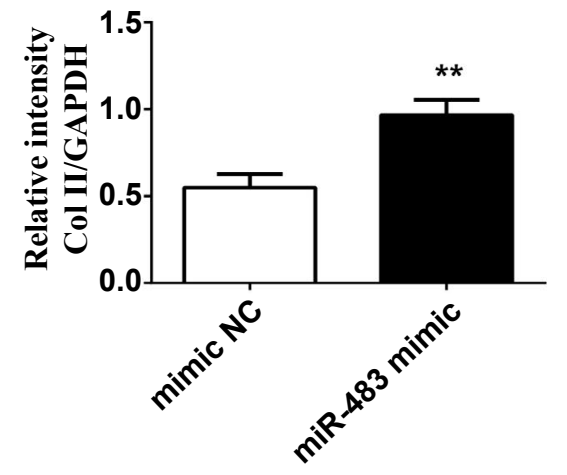

Fig. 2: MiR-483 improves the cell growth and inhibits the Col II expression

a. RT-PCR assay was used to determine the expression of miR-483. b. MTT assay was conducted to confirm the NP cell growth between miR-483 mimic and mimic NC groups (-०)miR-483 mimic, (-■-)mimic NC. c-d. western blot analysis was used to examine the expression of Col II. **, ***p<0.05
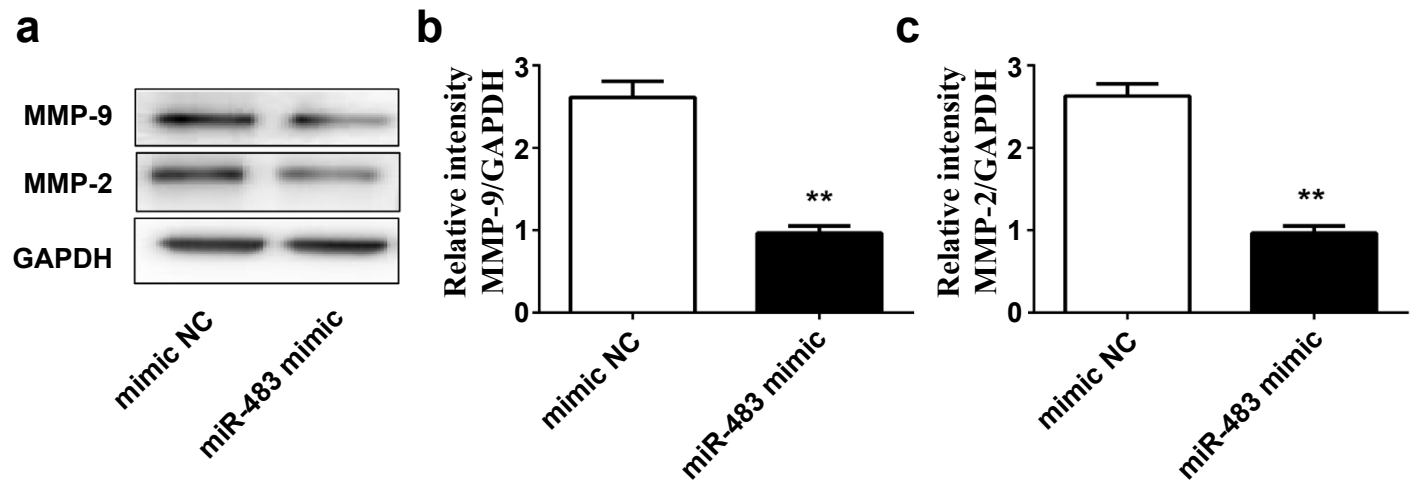

Fig. 3: MiR-483 increases the MMP-2 and MMP-9 expression

a-c. Western blot analysis was used to determine the MMP-2 and MMP-9 between miR-483 mimic and mimic NC groups. ${ }^{* *} \mathbf{p}<\mathbf{0 . 0 5}$.

it was also found that miR-483 could promote the expression of cyclin A and cyclin D1 (fig. 4).

In the present study, it was confirmed that the expression of miR-483 was downregulated significantly in IDD NP samples. Besides, miR-483 expression was negatively associated with the Pfirrmann grade. Furthermore, overexpression of miR-483 could promote NP cell growth, enhance the Col II expression as well as inhibit the MMP-2 and MMP-9 expression. In addition, miR-
483 caused an increase in the proportion of NP cells in the S-phase. These results indicated that miR-483 expression might play a vital role in the development and progression of IDD.

IDD is the pathological basis of common degenerative diseases in spinal surgery, which can increase patients' psychological burden, cause disability, reduce quality of life and increase economic burden ${ }^{[12]}$. Many factors have proven to be responsible for IDD, unfortunately, 
a

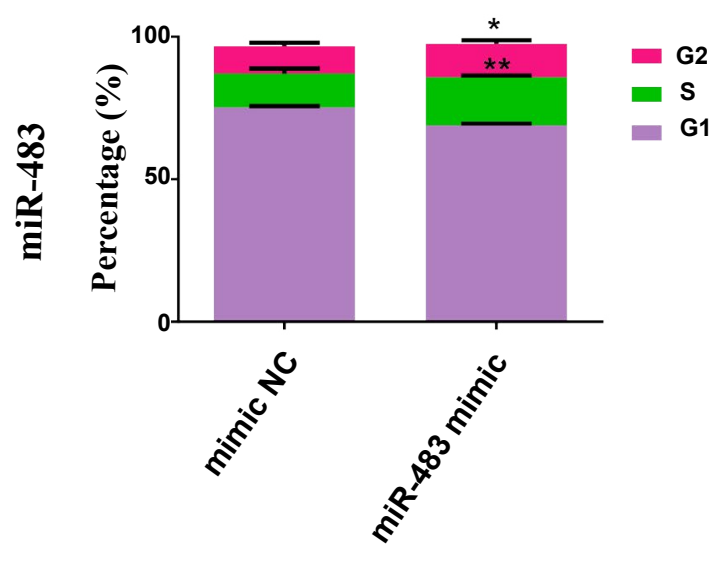

C

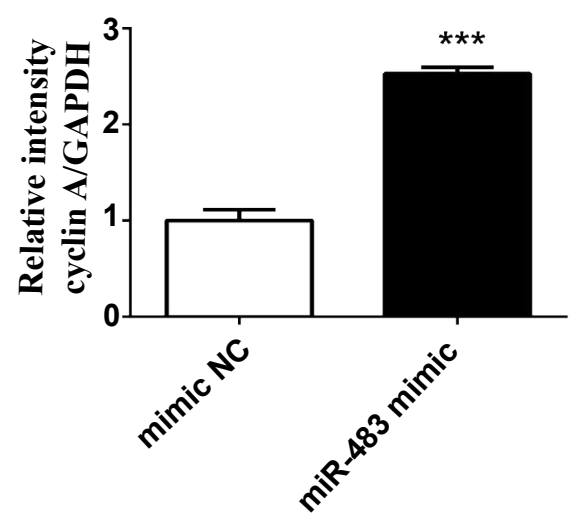

b

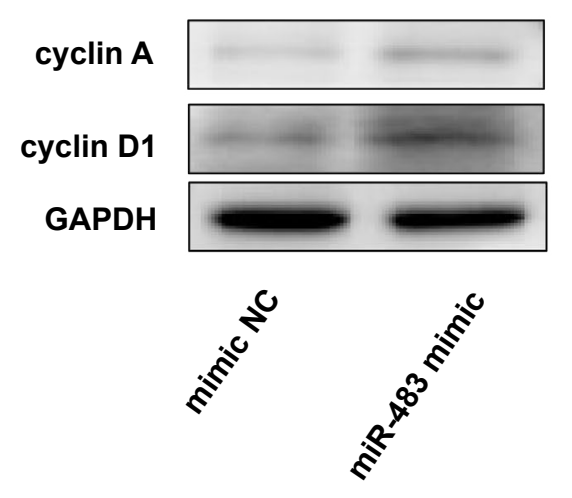

d

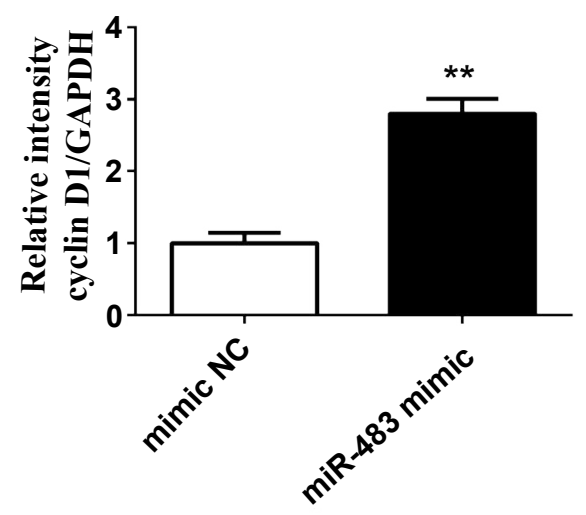

Fig. 4: MiR-483 regulates cell cycle and cyclin proteins a. Flow cytometry was conducted to detect the cell cycle information between miR-483 mimic and mimic NC groups. b. Western blot analysis was used to determine the cyclin A and cyclin D1 expression. *, **, *** p<0.05

the specific mechanism remained unclear ${ }^{[4]}$. NP cells exhibit changes in both phenotype and morphology with aging-related IDD that might impact the onset and progression of IDD pathology. The NP tissue, which located in the centre of intervertebral disc is composed of NP cells and $\mathrm{ECM}^{[13]}$. NP cells are involved in the synthesis and catabolism of ECM and excessive apoptosis of NP cells followed by the loss of ECM is considered to related to the development of $\operatorname{IDD}^{[6,7]}$. MiRNAs are related to the progress of many diseases and may become potential targets of treatment ${ }^{[8]}$. Interestingly, previous studies have showed that miRNAs play vital roles in the $\operatorname{IDD}^{[9,10]}$. For example, Ji et al. have indicated that microRNA-193a-3p was downregulated significantly in IDD and might exert its role by targeting MMP-14 ${ }^{[9]}$. In fact, previous microarray analysis showed that compared with control discs, miR-483 was downregulated significantly in IDD $^{[11]}$. However, whether miR-483 exerted biological functions in IDD remained unknown. The present study demonstrated that miR-483 expression was indeed downregulated in IDD NP samples and was negatively associated with the Pfirrmann grade. Considering that excessive apoptosis of NP cells was an important cause of IDD ${ }^{[6,7]}$, while the loss of Col II was an early indicator of $\operatorname{IDD}^{[14,15]}$, miR-483 mimic was used to further investigate the specific role of miR-483 in IDD, NP cell growth and on Col II expression in the present study. The data showed that miR-483 overexpression could promote NP cell growth and increase the Col II expression level in NP cells. These results suggested that excessive apoptosis of NP cells and reduced Col II caused by the downregulation of miR-483 might take part in the development of IDD. According to substrate specificity, MMP-2 and MMP-9 belonged to the gelatinises group of MMPs ${ }^{[16]}$. Recently, MMP-2 and MMP-9 expressions have suggested to be involved in the basement membrane collagen destruction and increased in degenerative disc disease ${ }^{[16,17]}$. The present study data showed overexpression of miR-483 could 
inhibit the expression of MMP-2 and MMP-9 in NP cells, which suggested that downregulation of miR-483 might participate in the development of IDD through MMP-2 and MMP-9 pathway.

As cyclin A reaches the highest level in S and G2 phases, it is considered to be a regulator of the transition to mitosis ${ }^{[18]}$. Whereas cyclin D1 is one of the most important mediators for cell transformation from G1 to S phase ${ }^{[19]}$. Thus, the effect of miR-483 on NP cells cycle was further investigated. Flow cytometry showed that miR-483 could increase the proportion of $\mathrm{S}, \mathrm{G} 2 / \mathrm{M}$ cells, while western blot indicated that miR483 could promote the expression of cyclin A and cyclin D1. These results demonstrated that miR-483 could promote DNA synthesis and mitosis of NP cells. In conclusion, it was found that miR-483 expression was significantly downregulated in IDD NP cases and negatively associated with the Pfirrmann grade. In addition, overexpression of miR-665 promoted NP cell growth, enhanced the Col II expression and inhibited the MMP-2 and MMP-9 expression. In addition, miR483 exerted an increase in the proportion of S-phase of NP cells. Taken together, this study revealed a novel miRNA in the progression of IDD, which could become a potential therapeutic approach for IDD.

\section{Conflict of interest:}

All authors report no conflicts of interest in this work.

\section{REFERENCES}

1. Feng G, Zhang Z, Jin X, Hu J, Gupte MJ, Holzwarth JM, et al. Regenerating nucleus pulposus of the intervertebral disc using biodegradable nanofibrous polymer scaffolds. Tissue Eng Part A 2012;18:2231-8.

2. Loreto C, Musumeci G, Castorina A, Loreto C, Martinez G. Degenerative disc disease of herniated intervertebral discs is associated with extracellular matrix remodelling, vimentinpositive cells and cell death. Ann Anat 2011;193:156-62.

3. McCann MR, Patel P, Pest MA, Ratneswaran A, Lalli G, Beaucage KL, et al. Repeated exposure to high-frequency low-amplitude vibration induces degeneration of murine intervertebral discs and knee joints. Arthritis Rheum 2015;67:2164-75.

4. Sun Z, Jian Y, Fu H, Li B. MiR-532 downregulation of the Wnt/beta-catenin signaling via targeting Bcl-9 and induced human intervertebral disc nucleus pulposus cells apoptosis. J Pharmacol Sci 2018;138:263-70.

5. David G, Ciurea AV, Iencean SM, Mohan A. Angiogenesis in the degeneration of the lumbar intervertebral disc. J Med Life 2010;3:154-61.

6. Liu J, Tao H, Wang H, Dong F, Zhang R, Li J, et al. Biological Behavior of Human Nucleus Pulposus Mesenchymal Stem Cells in Response to Changes in the Acidic Environment
During Intervertebral Disc Degeneration. Stem Cells Dev 2017;26:901-11.

7. Vadala G, Russo F, Di Martino A, Denaro V. Intervertebral disc regeneration: from the degenerative cascade to molecular therapy and tissue engineering. J Tissue Eng Regen $M$ 2015;9:679-90.

8. Sonkoly E, Pivarcsi A. Advances in microRNAs: implications for immunity and inflammatory diseases. J Cell Mol Med 2009; 13:24-38.

9. Ji ML, Zhang XJ, Shi PL, Lu J, Wang SZ, Chang Q, et al. Downregulation of microRNA-193a-3p is involved in invertebral disc degeneration by targeting MMP14. J Mol Med 2016;94:457-68.

10. Li W, Wang P, Zhang Z, Wang W, Liu Y, Qi Q. MiR-184 Regulates Proliferation in Nucleus Pulposus Cells by Targeting GAS1. World Neurosurg 2017;97:710-5 e1.

11. Sherafatian M, Abdollahpour HR, Ghaffarpasand F, Yaghmaei S, Azadegan M, Heidari M. MicroRNA Expression Profiles, Target Genes, and Pathways in Intervertebral Disk Degeneration: A Meta-Analysis of 3 Microarray Studies. World Neurosurg 2019;126:389-97.

12. Li G, Tang X, Chen H, Sun W, Yuan F. miR-148a inhibits pro-inflammatory cytokines released by intervertebral disc cells by regulating the p38/MAPK pathway. Exp Ther Med 2018;16:2665-9.

13. Pattappa G, Li Z, Peroglio M, Wismer N, Alini M, Grad S. Diversity of intervertebral disc cells: phenotype and function. J Anat 2012;221:480-96.

14. Rutges JP, Kummer JA, Oner FC, Verbout AJ, Castelein RJ, Roestenburg $\mathrm{HJ}$, et al. Increased MMP-2 activity during intervertebral disc degeneration is correlated to MMP-14 levels. J Pathol 2008;214:523-30.

15. Matsui Y, Maeda M, Nakagami W, Iwata $H$. The involvement of matrix metalloproteinases and inflammation in lumbar disc herniation. Spine 1998;23:863-8.

16. Goupille P, Jayson MI, Valat JP, Freemont AJ. Matrix metalloproteinases: the clue to intervertebral disc degeneration? Spine 1998;23:1612-26.

17. Kang JD, Stefanovic-Racic M, McIntyre LA, Georgescu HI, Evans $\mathrm{CH}$. Toward a biochemical understanding of human intervertebral disc degeneration and herniation. Contributions of nitric oxide, interleukins, prostaglandin E2, and matrix metalloproteinase. Spine 1997;22:1065-73.

18. Handa K, Yamakawa M, Takeda H, Kimura S, Takahashi T. Expression of cell cycle markers in colorectal carcinoma: superiority of cyclin A as an indicator of poor prognosis. Int $\mathrm{J}$ Cancer 1999;84:225-33.

19. Shimura T. Acquired radioresistance of cancer and the AKT/ GSK3beta/cyclin D1 overexpression cycle. J Radiat Res 2011;52:539-44.

This is an open access article distributed under the terms of the Creative Commons Attribution-NonCommercial-ShareAlike 3.0 License, which allows others to remix, tweak, and build upon the work non-commercially, as long as the author is credited and the new creations are licensed under the identical terms

This article was originally published in a special is-
sue, "Biomedical Research in Healthcare Setting"
Indian J Pharm Sci 2020:82(2)Spl issue5;1-5

